\title{
Vertical Alignment of Liquid Crytals on Polydimethylsiloxane Thin Films
}

\author{
W. Zheng, ${ }^{*}$ L.-H. Yang, and M.-C. Lee \\ Depatment of Photonics, National Sun Yat-Sen University, 70 Lienhai Road, Kaohsiung 80424, Taiwan, DOC.
}

Received February 10, 2011; accepted March 14, 2011; published March 31, 2011

\begin{abstract}
Studies on the use of polydimethylsiloxane to align liquid crystals have been carried out. The thin films prepared using the silicon-based elastomer, with a pretty low surface free energy, are found to be capable of vertically aligning liquid crystals. The pretilt angle of calamitic liquid crystals sandwiched between thin silicon elastomer films was virtually 90 degrees. Liquid crystal devices consisting of elastomer alignment layers can operate in the in-plane-switch mode.
\end{abstract}

A narrow viewing field is a knotty problem of liquid crystal displays (LCDs) which use calamitic liquid crystals (LCs) as optical media to modulate light. This problem originates from the anisotropic nature of calamitic liquid crystals that consist of rod-like molecules. In modern LCDs, a molecular ordering is required, in which nematic phase calamitic LC is aligned to have all molecules oriented in one direction and form a uniaxial optical wave plate. In the mentioned aligned LC medium, the optical axis is parallel to the average orientational direction, i.e. the director, of the LC. A light beam which is propagating in a direction deviated by an angle $\Theta$ from the director, will encounter a direction-dependent birefringence [1], and a propagating-direction-dependent retardation (PDDR) $\Delta \mathrm{n}(\Theta) d$ arises after it propagates a distance $d$ in the LC. The existence of the PDDR causes a viewing-direction-dependent degradation in optical performance, and leads to a narrow viewing field of the LCDs. Over the years, many new operating modes have been developed to increase the viewing angle of the LCDs. In addition, a vertical alignment liquid crystal (VALC), in which LC molecules in the sandwich-type cell are aligned to orient perpendicular to the substrates [2] emerges as one of the most effective modes which allow PDDR to be balanced in a wide viewing angle. VALC is now a leading technique used to fabricate wide-viewing-angle LCDs in the flat-panel-display industry [3].

Thereupon, materials that can promote VALC are extremely attractive. For many years, imide-based polymers have been widely used to achieve desired LC alignment, and polyimides that are capable of vertical alignment of LC are available. Although they are reliable, durable, easy in handling and cost effective, polyimides have some fatal weakness. Imide-based polymers can

\footnotetext{
*E-mail: wzheng@ymail.com
}

hardly match the requirements of the new generation flexible displays, and the toxic by-products during the PI process are harmful to the environment.

Polydimethylsiloxane (PDMS) is a silicon-based organic elastomer which has been widely used as a replica of microstructure that leads to a broad range of applications [4]-[6]. Efforts have also been made to exploit the use of PDMS in flexible LCDs [7]. In attempting to align liquid crystals, PDMS attracted attention for its high hydrophobicity. Recently, purposeful studies of the surface characteristics, in terms of energetic state, of PDMS films have been carried out. Here we demonstrate that PDMS thin films, with a pretty low surface free energy, may be a potential candidate for the commanding surfaces that support VALCs.

In our studies, the polymer precursor used was Sylgard 184 (Dow Corning), which is a mixture of base and curing agent in a ratio of base:curing agent $=10: 1$ by weight. PDMS thin films were produced by spin-casting the precursor on to glass plates, and then thermally curing the precursor layer in a temperature stable PE30/201 oven (Carbolite) at an elevated temperature $\left(>100{ }^{\circ} \mathrm{C}\right)$ for about $1 \mathrm{hr}$. In the cases of more precise control of sample temperature, a programmable temperature system Linkam TMS93 (Linkam Scientific Instrument Ltd) was used. After thermal curing, PDMS thin films, with a thickness within the 1.0-1.2 $\mu \mathrm{m}$ range, were formed on the glass substrates. As an LC alignment layer, the PDMS films are too thick. However, they are quite suitable for the surface characterisation of the elastomer.

The surface energetic states of the PDMS thin films were evaluated by means of the sessile drop method based on the Owens-Wendt model [8]. The measurements were carried out using a DSA100 drop shape analysis system (Krüss). The details of the measurements are described elsewhere [9]. The values of the surface free energy of the PDMS films were found to be within the range of $20-24 \mathrm{~mJ} / \mathrm{m}^{2}$. These values are much smaller than the surface free energy $\left(31 \mathrm{~mJ} / \mathrm{m}^{2}\right)$ of typical PI thin films produced by using SE-4811 (Nissan Chemical), which is a commercial PI resin used to produce VA layers in the LCD industry. 
The curing temperature was found to influence the surface free energy of the resultant PDMS films. Figure 1 shows the surface free energy of the PDMS film as a function of the curing time for different curing temperatures. When curing the precursor at $100^{\circ} \mathrm{C}$, the PDMS film will take more than one hour to reach the stable energetic state and the resultant elastomer film exhibits a surface free energy above $24 \mathrm{~mJ} / \mathrm{m}^{2}$. An increase in the curing temperature can shorten the curing time, and also result in a decrease in the surface free energy of the resultant PDMS film. When the curing temperature was increased to $130^{\circ} \mathrm{C}$, the surface free energy of the PDMS, as illustrated in Fig. 1, can reach a value below $22 \mathrm{~mJ} / \mathrm{m}^{2}$ after being cured for $40 \mathrm{~min}$. The temperature-dependent conversion rate of the precursor has not been examined yet at this stage of the present study. The curing-temperature-dependent surface free energy is believed, however, to result from an incomplete conversion of precursor and/or different morphologies of the resultant PDMS thin films.

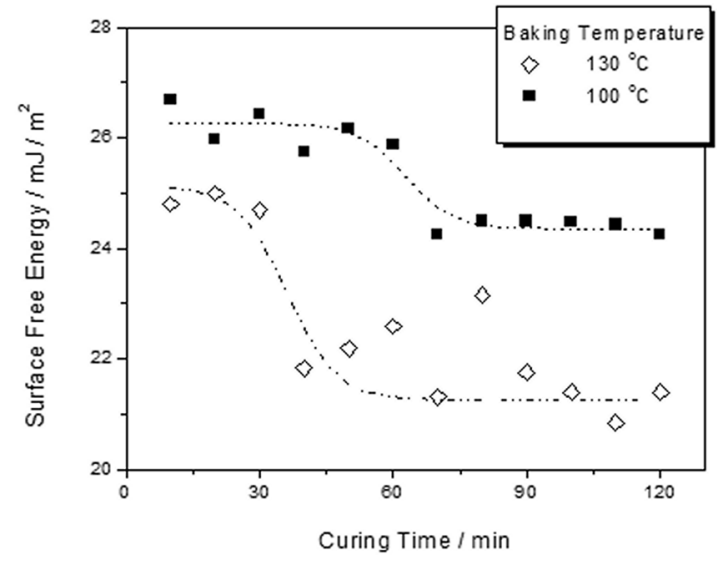

Fig. 1. Surface energy as a function of curing time for the PDMS films cured at $100^{\circ} \mathrm{C}$ and $130^{\circ} \mathrm{C}$, respectively.

Although the correlation between surface energy and molecular alignment has not been clearly elucidated yet, it is accepted that the surface with low surface free energy will support VA of LC molecules [10]-[11]. The low surface free energy of the PDMS films allows us to expect the elastomer to be capable of aligning LC vertically. Indeed, the pretilt angle of the LC molecules against the surface of the PDMS film, measured for E7 and MLC7026-100 (both are from E. Merck), was found to be virtually $90^{\circ}$, and remained unchanged when the surface free energy of the PDMS film was increased up to 30 $\mathrm{mJ} / \mathrm{m}^{2}$ (Fig. 2). In a polarising microscope, the appearances of the LC layers sandwiched between PDMS thin films, which are not shown here, were completely black, and did not vary when the samples were rotated. It is then confirmed that the LC molecules in the samples are orienting perpendicular to the surface.
The electrooptical characterisation of LC devices consisting of PDMS alignment layers was carried out using sandwich-type samples. For this purpose, empty cells were made by putting two PDMS coated substrates together with PDMS surfaces facing each other. The gap between two substrates for all cells used in the present study was fixed at $10 \mu \mathrm{m}$.

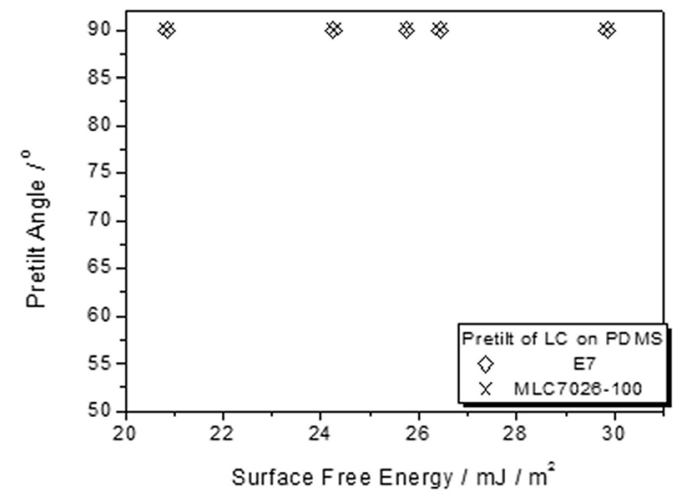

Fig. 2. Pretilt angles of nematic LC molecules on the PDMS surface against surface free energy.

Both positive and negative electrodes, each of which consists of a group of $4 \mu \mathrm{m}$ wide ITO conducting stripes distributed equidistantly in one-dimension with a spatial period $30 \mu \mathrm{m}$ and in a comb-type formation, are mounted on the same substrate (cf. Fig. 3). Therefore, the LC in the cells will operate in the in-plane-switching (IPS) mode. The LC molecules were aligned to tilt a small angle, i.e. the pretilt angle, away from the normal to the substrate in order to regulate the reorientation of the LC molecules and allow the LC to operate properly in IPS mode.
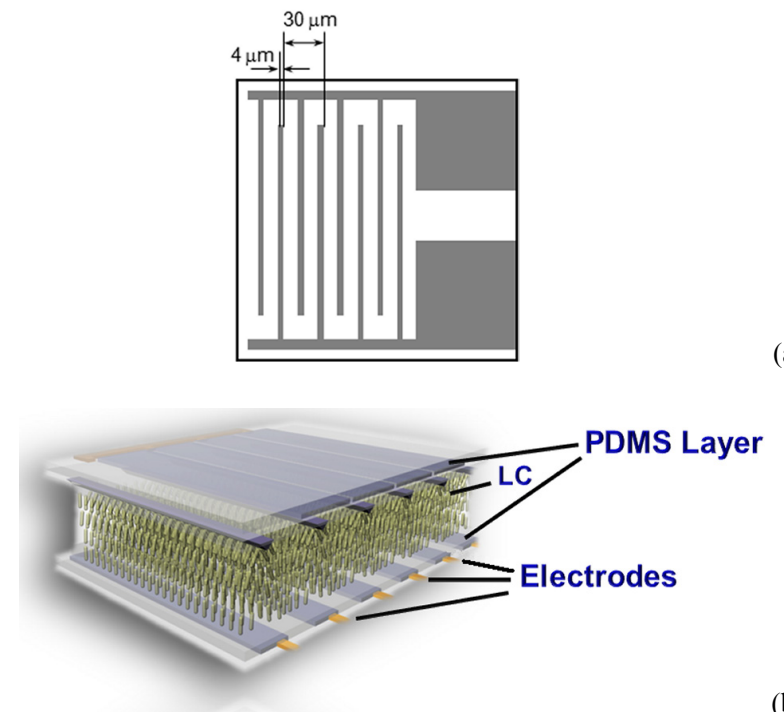

(b)

Fig. 3. (a) The layout of the electrodes on the electrode-substrate. (b) Schematic diagram showing the in-plane-switch LC cell, in which protuberances have been produced on one of the substrates, used in the present studies. 
For this purpose, protuberances were produced on one of the two substrates of the cell using a thermal-induced-deformable photoresist and subsequently covered by PDMS thin film [12]. Although we were unable to determine precisely the pretilt angle, the electrooptic measurement revealed that the device operated very well.

The nematic mixture E7 was injected into the cells in the isotropic phase at $100^{\circ} \mathrm{C}$. In the field-off state, the LC molecules are aligned perpendicular to the substrates [Fig. 4(a)], and no light can propagate across the device. Therefore, the sample, as illustrated in Fig. 4(c), exhibits a black appearance. Upon applying an external field, the molecules were realigned [Fig. 4(b)] and disorder in the molecular assembly in the cell arose, leading to light leakage from the device and thus a white appearance of the sample [Fig. 4(d)]. The threshold voltage was measured to be $10.2 V_{\text {rms }}$. The total response time, defined as the sum of the rise and the relaxation times corresponding to field on and off states, respectively, was $234 \mathrm{~ms}$.

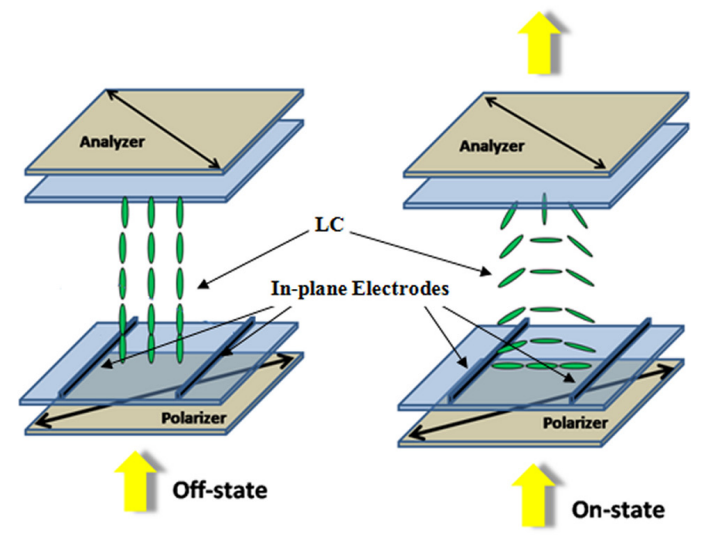

(a)

(b)

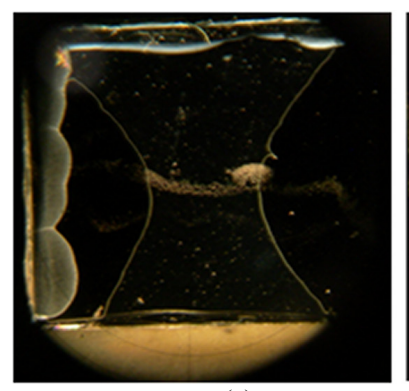

(c)

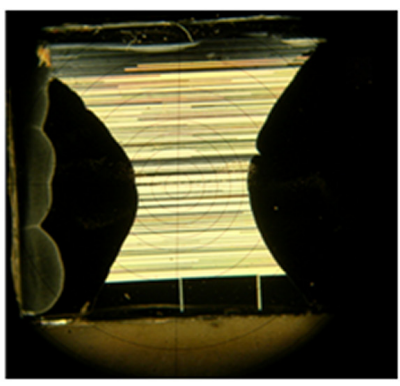

(d)

Fig. 4. Schematic showing of the orientational states of liquid crystal molecules in the test cell, with PDMS as an alignment layer in (a) the field-off and (b) the field-on states, respectively. Photographs of the sample show (c) the black and (d) white states corresponding to the field-off and field-on states, respectively.
The large threshold and the slow response speed of the sample was expected as E7 is not suitable for this type of device. We believe that better performance can be achieved when the right LCs are selected and the geometrical parameters of the cell, such as the gap between electrodes, thickness of the PDMS film and the thickness of the cell, etc., are optimized.

In conclusion, we have studied the possibility of using PDMS as a commanding layer to achieve vertical alignment of LCs. Surfaces coated with a thin layer of PDMS exhibit very low surface free energy, and are found to be capable of vertically aligning calamitic LCs. The LC devices consisting of a PDMS alignment layer can operate in the IPS mode. Considering its low toxicity and easy processing, the silicon-based organic elastomer is a promising material for new generation display technology and relevant photonic device applications. Further studies are being carried out to exploit the usefulness of the elastomer in fabricating flexible display devices.

This work is funded by the National Science Council of the Republic of China (NSC99-2221-E110-056).

\section{References}

[1] E. Lueder, Liquid Crystal Displays-Addressing Schemes and Electro-Optical Effects (Chichester, John Wiley \& Sons 2001).

[2] J. Glueck, (PhD Thesis, University of Stuttgart 1995).

[3] DisplaySearch, Quarterly Wide Viewing Angle Technology Report (2010),http://www.displaysearch.com/cps/rde/xchg/diaplaysearch/hs.X sl/quarterly wide viewing angle technology shipment and forecast database.asp

[4] C.M. Knobler, Nature 369, 15 (1994).

[5] D. Qin, Y. Xia, G.M. Whitesides, Adv. Mater. 8, 917 (1996).

[6] M. Geissler, Y. Xia, Adv. Mater. 16, 1249 (2004).

[7] Y.T. Kim, J.H. Hong, T.Y. Yoon, S.D. Lee, Appl. Phys. Lett. 88, 263501 (2006).

[8] D.K. Owens, R.C. Wendt, J. Appl. Polym. Sci. 13, 1741 (1969).

[9] W. Zheng, C. H. Lu, Y. Yeh, Jpn. J. Appl. Phys. 47, 1651 (2008)

[10] L.T. Creagh, A.R. Kmetz, Mol. Cryst. Liq. Cryst. 24, 59 (1973).

[11] F.J. Kahn, C.N. Tayor, H. Schonhorn, Proc. IEEE 61, 823 (1973)

[12] W. Zheng, L. Yang, R.O.C. Patent, 200931140. 